\title{
Cell attachment and proliferation of osteoblast-like MG63 cells on silk fibroin membrane for guided bone regeneration
}

\author{
Chae-Kyung Yoo ${ }^{1+}$, Jae-Yun Jeon ${ }^{2+}$, You-Jin Kim³ , Seong-Gon Kim ${ }^{4}$ and Kyung-Gyun Hwang ${ }^{1,2^{*}}$
}

\begin{abstract}
Background: The aim of this study is to verify the feasibility of using silk fibroin (SF) as a potential membrane for guided bone regeneration (GBR).

Methods: Various cellular responses (i.e., cell attachment, viability, and proliferation) of osteoblast-like MG63 cells cultured on an SF membrane were quantified. After culturing on an SF membrane for 1, 5, and 7 days, the attachment and surface morphology of MG63 cells were examined by optical and scanning electron microscopy (SEM), cell viability was determined using a 3-(4,5-dimethylthiazol-2-yl)-2,5-diphenyltetrazolium bromide (MTT) assay, and cell proliferation was quantified using 4',6-diamidino-2-phenylindole (DAPI) fluorescence staining.

Results: Optical microscopy revealed that MG63 cells cultured on the SF membrane proliferated over the 7-day observation period. The viability of cells cultured on SF membranes (SF group) and on control surfaces (control group) increased over time $(P<0.05)$; however, at respective time points, cell viability was not significantly different between the two groups $(P>0.05)$. In contrast, cell proliferation was significantly higher in the SF membrane group than in the control group at 7 days $(P<0.05)$.
\end{abstract}

Conclusions: These results suggest that silk fibroin is a biocompatible material that could be used as a suitable alternative barrier membrane for GBR.

Keywords: Bone regeneration, Cell adhesion, Cell proliferation, Membrane, Osteoblasts, Silk fibroin

\section{Background}

Several approaches for alveolar bone regeneration have been studied, including autogenous graft, allogenic graft, xenogenic graft, use of alloplastic materials, and distraction osteogenesis [1-3]. Guided bone regeneration (GBR) was introduced in the context of orthopedic research as early as 1959 [4]. The basic principles of GBR as a surgical procedure were developed by Melcher in 1976 and involve suppressing the growth of unwanted cellular tissues to create space for the growth of the desired tissue [5]. Features of an ideal membrane include

\footnotetext{
*Correspondence: hkg@hanyang.ac.kr

'Equal contributors

'Department of Translational Medicine, Graduate School of Biomedical Science and Engineering, Hanyang University, Seoul, South Korea

${ }^{2}$ Department of Dentistry/Division of Oral and Maxillofacial Surgery, College of Medicine, Hanyang University, 222 Wangsimni-ro, Seongdong-gu, Seoul 133-791, South Korea

Full list of author information is available at the end of the article
}

easy handling, bio-absorbability, and biofunctionality [6, 7]. Membranes could additionally prevent epithelial cell movement to the bone defect site while permitting osteoblast migration to allow osteoblasts to carry out the regenerative process $[8,9]$. Therefore, ideal membranes used in bone tissue regeneration should prevent local tissue cells of the sintered body and other epithelial cells from accessing the bone defect area [10, 11]. However, currently developed resorbable membranes are unable to achieve complete bone regeneration because of an induced inflammatory response caused by inadequate micro-environmental separation $[12,13]$. Therefore, the development of a biocompatible membrane that resolves these current limitations is important and necessary.

Non-woven silk fibroin net is produced from the silk cocoon of the common silkworm moth, Bombyx mori. Silk fibroin (SF) has the ability to support the growth of different cell types, including endothelial, epithelial, 
fibroblast, glial, keratinocyte, and osteoblast cells [14]. It has also been applied in various biomedical applications: as a substrate material for tissue engineering scaffolds, in drug delivery, and even as artificial blood vessels because of its high tensile strength and low solubility in aqueous solutions $[15,16]$. The physical and biological properties of silk fibroin make it an ideal candidate material for barrier membranes that resolve the limitations of previously developed membranes $[17,18]$.

Thus, the aim of this study is to evaluate various cellular responses of osteoblast-like MG63 cells to silk fibroin to verify the effectiveness and feasibility of silk fibroin as a potential membrane for GBR.

\section{Methods}

\section{Cell culture on SF membranes}

Osteoblast-like MG63 cells (ATCC, Manassas, VA, USA) were cultured 100-mm culture dishes ingrown in Dulbecco's modified Eagle medium (DMEM, Gibco, USA) supplemented with $10 \%$ fetal bovine serum (FBS, ATLAS, Dae Myung Science Co., Ltd., Korea) and $1 \%$ penicillin-streptomycin (Pen-Strep, Gibco, USA). Cultures were incubated at $37{ }^{\circ} \mathrm{C}$ in a humidified atmosphere of $95 \%$ air, and $5 \% \mathrm{CO}_{2}$, and the medium was changed every 2 days [19].

SF membranes were prepared as previously described and stored in a sterile environment. Membranes were cut into 5 -mm-diameter discs and sterilized by immersion in $70 \%$ ethanol for $10 \mathrm{~min}$ followed by rinsing with phosphate-buffered saline (PBS). Sterile discs were placed in 24-well culture plates with spray glue, and culture medium was added to keep discs moist until cells were seeded. MG63 cells, cultured in 100-mm cell culture dishes, were trypsinized (0.25\% trypsin EDTA, Gibco, USA), and $3 \times 10^{4}$ cells in $1 \mathrm{~mL}$ of fresh medium were seeded onto 24-well plates with or without prepared SF membranes (SF membrane group or control group, respectively; Fig. 1).

\section{Visualization of cell attachment}

After culturing for $0,1,5$, or 7 days, attached cells were imaged with optical microscopy (Leica DMI4000B, Leica, Germany) and scanning electron microscopy (SEM, Nova NanoSEM 450, FEI, USA) to compare the morphology of cells on the surface of SF membranes (SF membrane group) to those on culture plastic (control group). SEM imaging was performed after fixing cells with $4 \%$ paraformaldehyde (Sigma-Aldrich, Inc., USA), dehydrating samples with graded ethanol (EMSURE ${ }^{\oplus} \mathrm{Ph}$ Ethanol absolute for analysis) (70, 90, and $100 \%$ ), and further drying samples in an oven for $24 \mathrm{~h}$. Fixed samples were sputter-coated with gold and imaged with the SEM [20].

\section{MTT assay for cell viability}

The viability of cells cultured on SF membranes for various times up to 7 days was evaluated using thiazolyl blue tetrazolium bromide (MTT, Sigma-Aldrich, Inc., USA) following the manufacturer's instructions. MTT reagent was added to each sample and incubated for $3 \mathrm{~h}$ to allow the formation of MTT formazan. The resulting formazan was educed with dimethyl sulfoxide (DMSO, Sigma-Aldrich, Inc., USA), and the absorbance of each solution was measured at a wavelength of $595 \mathrm{~nm}$ with a microplate reader (Bio-Rad, Japan) in triplicate. Cell viability was determined by comparing the absorbance of samples to a standard curve [21].

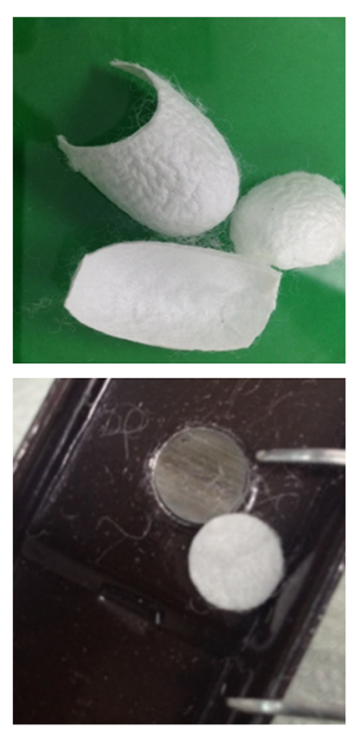

24-well culture plate
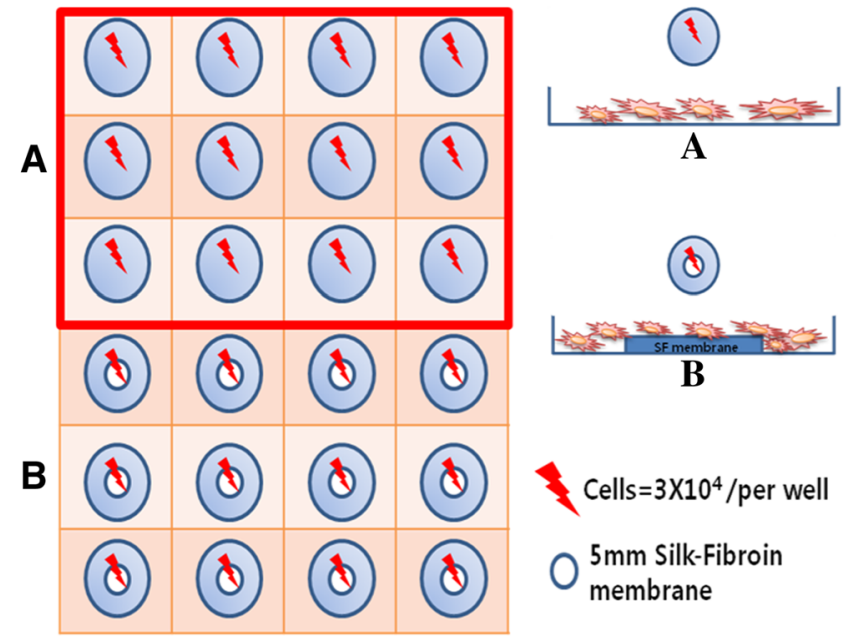

A

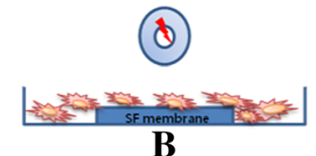

Cells $=3 \times 10^{4} /$ per well

$5 \mathrm{~mm}$ Silk-Fibroin

membrane

Fig. 1 Silk membranes were prepared for culture with cells by cutting the film into discs $5 \mathrm{~mm}$ in diameter. Osteoblast-like MG63 cells $\left(3 \times 10^{4}\right.$ cells $/ \mathrm{mL}$ ) was seeded onto a new 24-well plate with $1 \mathrm{~mL}$ fresh medium to each well. a Control group; $\mathbf{b}$ silk fibroin membrane group 
DAPI staining for counting cell numbers

The number of cells was determined by 4',6-diamidino-2-phenylindole (DAPI) (Vector Laboratories, Inc., Burlingame, CA, USA) fluorescence staining. Cells grown on SF membranes were washed with PBS (Gibco, USA) once before and twice after being fixed on the membrane with $4 \%$ paraformaldehyde (Sigma-Aldrich, Inc., USA) for $15 \mathrm{~min}$ at room temperature. Membranes were then mounted on a slide, and the nuclei of cells were stained with DAPI and visualized and counted using an inverted fluorescence microscope (Leica DMI4000B, Leica, Germany) [22]. Visual field for cell counting was selected randomly.

\section{Statistical analysis}

Each experiment was conducted at least thrice and replicated four times. All the data were expressed as the mean \pm standard deviation for $n=4$. The statistical difference was analyzed using Kruskal-Wallis one-way analysis of variance by ranks (IBM SPSS Statistics 21, IBM, USA), and a $P$ value of $<0.05$ was considered significant.

\section{Results}

Cell attachment and proliferation on SF membranes

MG63 cells were seeded onto SF membranes (SF membrane group) or on culture plastic (control group) and assessed for differences in their proliferation over a range of 1 to 7 days (Fig. 2). MG63 cells were still in suspension $1 \mathrm{~h}$ after seeding; however, after 1 day, cells had adhered onto or grew adjacent to the membrane (Fig. 2a). After 5 days, cells were starting to form observable colonies (Fig. 2b), and after 7 days, colonies were larger and denser (Fig. 2c).

\section{Cell confluence on SF membranes}

After culturing MG63 cells on SF membranes for 0, 1, 5, or 7 days, membrane surfaces were imaged by SEM to determine cell confluence (Fig. 3). After 1 day, MG63 cells were $10-20 \%$ confluent on the SF membrane surface compared with 0 day (Fig. 3a, b). After 5 days, MG63 cells were 50-60\% confluent (Fig. 3c), and by day 7 , cells were $90 \%$ confluent, nearly covering the entire surface of the membrane (Fig. 3d).

\section{Cell viability on SF membranes}

Though the optical density values of formazan solutions from the SF membrane group were lower than those from the control group at respective time points (Fig. 4), these differences were not statistically significant $(P>0.05)$. This is consistent with our data that the proliferation of cells in the SF membrane group was similar to that of the control group at 7 days.

\section{Counting cell number on SF membranes}

To compare proliferation rates, the number of cells on SF membranes (SF membrane group) or on culture plastic (control group) was quantified over time by counting DAPI-stained nuclei (Fig. 5). We quantified the average number of cells in at least 10 photomicrographs, which were captured at various regions of the SF membrane including from the periphery to the center (Fig. 6). On day $0,2.8$-fold more cells adhered to the culture plastic (control group, $344 \pm 180$ cells) than to SF membranes (SF membrane group, $123 \pm 33$ cells). After 1 and 5 days, the fold difference in the number of cells between the control and SF membrane group was only 1.1, although the control group still had a greater number of cells. After 7 days, 1.6-fold more cells were counted in the SF membrane group $(9821 \pm 3351)$ than in the control group $(6095 \pm 848)$. Although the number of cells in both groups increased significantly over time $(P<0.05)$, differences in the number of cells between the control and SF membrane group were not significantly different $(P>0.05)$ at 0,1 , and 5-day points. The number of cells in the control and SF membrane group increased by 17.7 and 79.8 times, respectively, over 7 days $(P<0.05)$. Thus, cells proliferated 4.5 -fold faster in the SF membrane group than in the control group, and the number
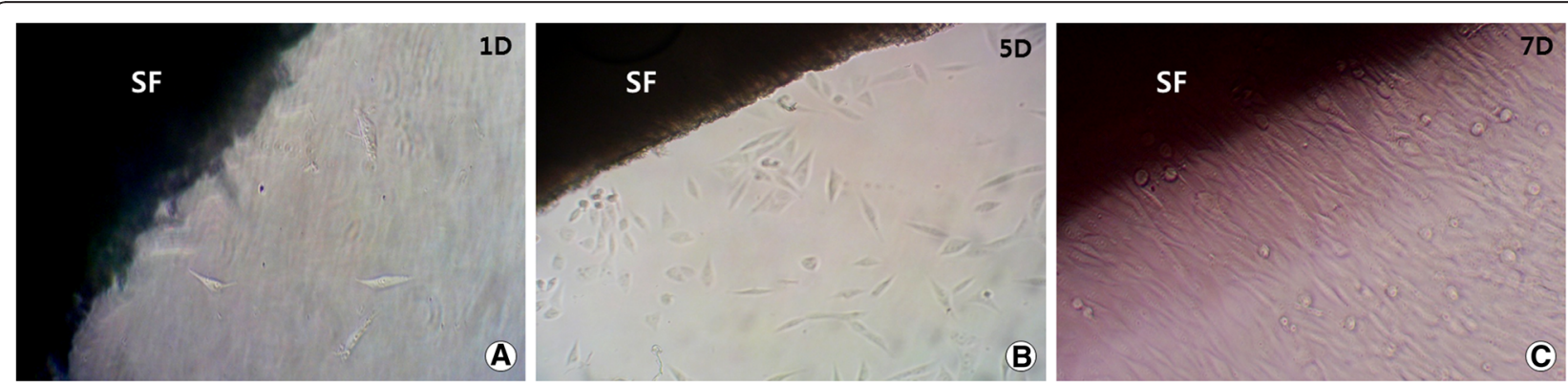

Fig. 2 Cells multiplied and proliferated around the silk fibroin membrane from 1 to 7 days. a At day 1 , the attachment of the cells to one or two peripheral membranes was confirmed. b At 5 days, the cells were attached around the membrane and had formed colonies. c At 7 days, the cells had proliferated and attached around the membrane to confluence (Optika $\times 10 / 0.25)$. SF silk fibroin membrane, 1D 1 day after cell seeding, 5D 5 days after cell seeding, 7D 7 days after cell seeding 


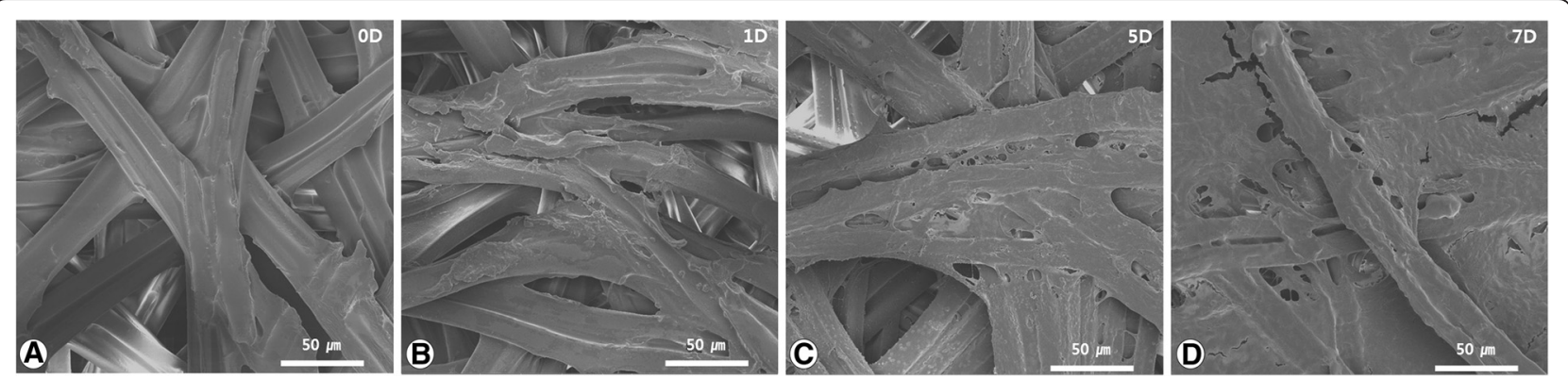

Fig. 3 Scanning electron microscopy (SEM) images of cell attachment on the surface of the silk fibroin (SF) membrane. a SEM image shows the SF membrane surface for day 0 , after seeding the initial cell number of $3 \times 10^{4}$. $\mathbf{b}$ The cells gradually filled across the fiber texture covering approximately 10-20\% of the SF membrane surface. c At 5 days, the gap between the SF membrane fibers was filled and covered by the cell attachment. d At 7 days, the surface of the SF membrane was nearly filled and covered with cells, to about $90 \%$ confluence, and the fiber texture was nearly totally obscured in the SEM image. OD cell seeding day, 1D 1 day after cell seeding, 5D 5 days after cell seeding, 7D 7 days after cell seeding

of cells in the SF membrane group was significantly increased more than that in the control group at 7 days $(P<0.05)$.

\section{Discussion}

In this study, we validated the biocompatibility of silk fibroin by showing that osteoblast-like MG63 cells can attach to, are viable on, and can proliferate on SF membranes. Silk fibroin is not only easily obtained from the common silkworm cocoon, but it is also recognized for its superior biocompatibility [23]. Osteogenic cells migrate to defective alveolar bone regions as part of the regenerative process, where a semi-permeable barrier membrane may assist in regulating the passage of specific biomolecules, such as growth factors that support angiogenesis, cytokines, and other nutrients [19, 24].

Attachment of MG63 cells onto the surface of SF membranes could not be verified by optical microscopy. However, the proliferation of cells adjacent to the membrane was confirmed. Additionally, adherent cells on SF membranes were observed by SEM analysis, which

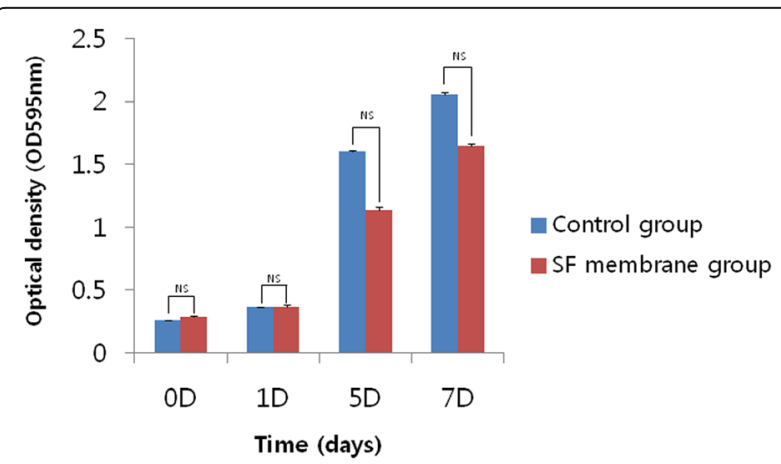

Fig. 4 Evaluation of the cell viability by MTT assay using the optical density value. OD optical density, SF silk fibroin, OD cell seeding day, 1D 1 day after cell seeding, 5D 5 days after cell seeding, 7D 7 days after cell seeding, NS not significant agrees with previous results. Kim et al. [18] showed the attachment of a confluent layer of cells on SF membranes with SEM, and Minoura et al. [25] confirmed the ability of silk fibroin nets to promote cellular attachment and growth with confocal microscopy and SEM.

Previous studies have also investigated the interaction of different cells with different membranes. Berahim et al. [26] observed the attachment and proliferation of fibroblasts on commercial collagen- and polyglycolic acidbased membranes with SEM over time. Carpio et al. [27] compared the attachment of cells on resorbable membranes (collagen membrane, glycolide fiber membrane) versus non-resorbable polytetrafluoroethylene (ePTFE) membranes with SEM. Approximately two times more cells attached onto the resorbable membrane than ePTFE. Additionally, a similar study by Wang et al. [28], in which they imaged osteoblast cells on six commercial membranes (BioMend, Resolut, GUIDOR, Epi-Guide, Gore-Tex, and Millipore filter) over time with SEM, had similar results as ours. Thus, silk fibroin has comparable cell attachment properties as other commercially manufactured membranes.

We investigated the proliferation and viability of MG63 cells on SF membranes using MTT assays. The growth rate of cells in the two groups was not significantly different $(P>0.05)$. Previous studies, which also used MTT assays, demonstrated that cell viability and proliferation were promoted by silk fibroin [18, 29]. Using MTT assays and measuring ALP activity, Cai et al. [30] showed that the proliferation of cells on silk fibroin was equal to or better than that of cells on other membranes tested. This is consistent with our results, which show that SF membranes do not negatively influence cell proliferation and viability.

The MTT assay could not be used to count the number of membrane-attached cells directly. SEM imaging was performed to demonstrate cell attachment, but it 


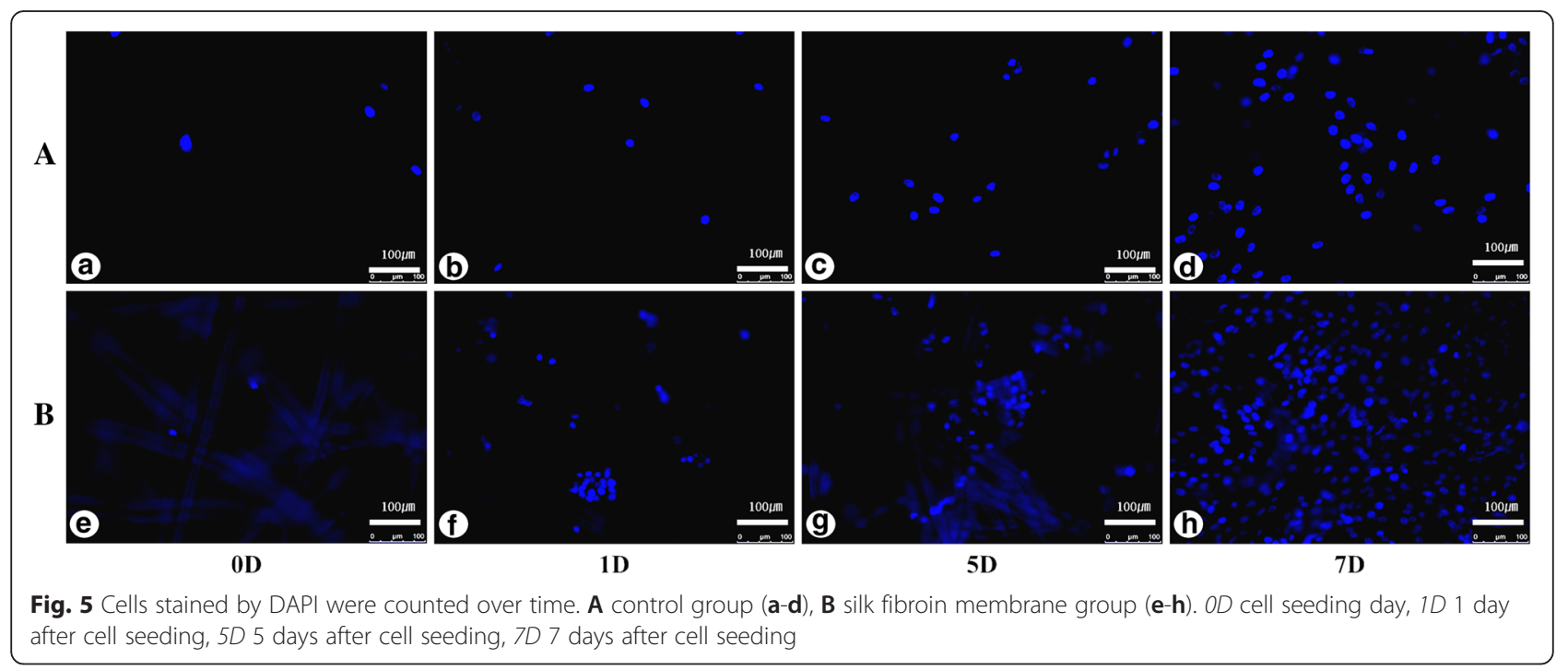

also could not be used to quantify the number of cells over time. Therefore, the number of cells was quantified by counting the number of DAPI-stained nuclei at various time points, which confirmed the proliferation of cells on SF membranes [31]. The number of cells in the SF membrane group counted after 7 days was greater with the DAPI-stained nuclei than by MTT assay. Whereas cells in the entire well (i.e., on and surrounding SF membranes) were included in MTT assays, only cells adhered onto SF membranes were included in the quantification of DAPI-stained nuclei.

SF membranes were prepared by first selecting silk fibroin following methods described in a previous study [29]. Although silk fibroin is water-absorbent, SF membranes will first float since considerable time is required for its saturation. Thus, we tested methods to adhere silk fibroin onto a 12-mm cover glass to resolve this issue.

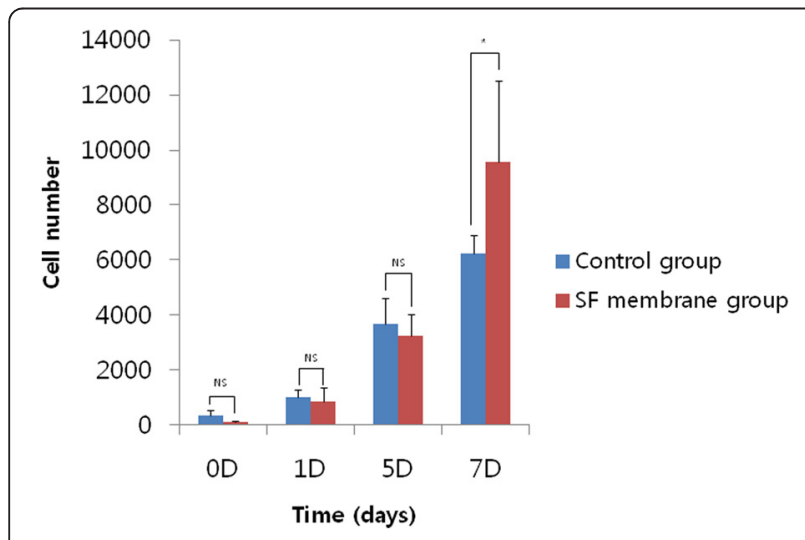

Fig. 6 Comparing the number of cells between the both groups through DAPI staining. SF silk fibroin, OD cell seeding day, 1D 1 day after cell seeding, 5D 5 days after cell seeding, $7 D 7$ days after cell seeding, NS not significant. The asterisk means significant at $P<0.05$
Double-sided tape has been used to affix SF membranes onto plates [21]; in that case, cell attachment is compromised due to the barrier of the rings and the thickness of the tape. Therefore, spray glue was used to affix SF membranes to the cover glass.

Silk fibroin has favorable biocompatibility, oxygen permeability, and cell attachment capabilities and can be provided cheaply through industrial manufacturing [17, 32, 33]. SF membranes are suitable for bone regeneration process because they can promote an osteoblast response with appropriate calcium deposition and nodule formation in vitro [34], and Sofia et al. [35] evaluated the use of silk fibroin for new bone regeneration in vivo in an animal study. On the basis of our results and previous studies, silk fibroin represents a comparable or better material for use as a barrier membrane for biomedical applications, including for guided bone regeneration.

\section{Conclusions}

Within the limits of our results, it was confirmed that the silk fibroin had a good biocompatibility about cell attachment and proliferation. This study suggests that silk fibroin membrane would be useful as a barrier material for GBR.

\section{Abbreviations \\ GBR: guided bone regeneration; SEM: scanning electron microscope; SF: silk fibroin.}

\section{Competing interests}

The authors declare that they have no competing interests.

\section{Authors' contributions}

CK did most experiments. KG and SG designed this experiment and reviewed this manuscript. CK, JY, and $Y J$ analyzed the data and performed the statistical analysis and wrote the manuscript. All authors read and approved the final manuscript. 


\section{Acknowledgements}

1. This work was supported by the National Research Foundation of Korea (NRF) grant funded by the Korean government (NRF-2013R1A1A2009975).

2. This work was supported by a grant from the Next-Generation BioGreen 21 Program (No.PJ01121404), Rural Development Administration, Republic of Korea.

\section{Author details}

'Department of Translational Medicine, Graduate School of Biomedical Science and Engineering, Hanyang University, Seoul, South Korea. ${ }^{2}$ Department of Dentistry/Division of Oral and Maxillofacial Surgery, College of Medicine, Hanyang University, 222 Wangsimni-ro, Seongdong-gu, Seoul 133-791, South Korea. ${ }^{3}$ Korean Minjok Leadership Academy, Hoengseong, South Korea. ${ }^{4}$ Department of Oral and Maxillofacial Surgery, College of Dentistry, Gangneung-Wonju National University, Gangneung, South Korea.

\section{Received: 21 January 2016 Accepted: 10 March 2016}

\section{Published online: 30 March 2016}

\section{References}

1. McAllister BS, Haghighat K (2007) Bone augmentation techniques. J Periodontol 78(3):377-396

2. Caplanis N, Sigurdsson TJ, Rohrer MD, Wikesjö U (1996) Effect of allogeneic, freeze-dried, demineralized bone matrix on guided bone regeneration in supra-alveolar peri-implant defects in dogs. Int J Oral Maxillofac Implants 12(5):634-642

3. Massaro D, Massaro GD (2004) Estrogen regulates pulmonary alveolar formation, loss, and regeneration in mice. Am J Phys Lung Cell Mol Phys 287(6):L1154-L1159

4. Hurley LA, Stinchfield FE, Bassett A, Lyon WH (1959) The role of soft tissues in osteogenesis. J Bone Joint Surg Am 41:1243-1266

5. Melcher A (1976) On the repair potential of periodontal tissues. J Periodontol 47(5):256-260

6. Kansy M, Senner F, Gubernator K (1998) Physicochemical high throughput screening: parallel artificial membrane permeation assay in the description of passive absorption processes. J Med Chem 41(7):1007-1010

7. Yuan J, Liu X, Akbulut O, Hu J, Suib SL, Kong J, Stellacci F (2008) Superwetting nanowire membranes for selective absorption. Nat Nanotechnol 3(6):332-336

8. Griffith LG, Naughton G (2002) Tissue engineering - current challenges and expanding opportunities. Science 295(5557):1009-1014

9. Schenk RK, Buser D, Hardwick WR, Dahlin C (1994) Healing pattern of bone regeneration in membraneprotected defects: a histologic study in the canine mandible. Int J Oral Maxillofac Implants 12(6):844-852

10. Zitzmann NU, Naef R, Schärer P (1996) Resorbable versus nonresorbable membranes in combination with Bio-Oss for guided bone regeneration. Int J Oral Maxillofac Implants 12(6):844-852

11. Fujihara K, Kotaki M, Ramakrishna S (2005) Guided bone regeneration membrane made of polycaprolactone/calcium carbonate composite nano-fibers. Biomaterials 26(19):4139-4147

12. Roccuzzo M, Bunino M, Needleman I, Sanz M (2002) Periodontal plastic surgery for treatment of localized gingival recessions: a systematic review. J Clin Periodontol 29(s3):178-194

13. Simion M, Scarano A, Gionso L, Piattelli A (1996) Guided bone regeneration using resorbable and nonresorbable membranes: a comparative histologic study in humans. Int J Oral Maxillofac Implants 11(6):735-742

14. Xia Q, Zhou Z, Lu C, Cheng D, Dai F, Li B, Zhao P, Zha X, Cheng T, Chai $C$ (2004) A draft sequence for the genome of the domesticated silkworm (Bombyx mori). Science 306(5703):1937-1940

15. Hutmacher DW (2000) Scaffolds in tissue engineering bone and cartilage. Biomaterials 21(24):2529-2543

16. Lutolf M, Hubbell J (2005) Synthetic biomaterials as instructive extracellular microenvironments for morphogenesis in tissue engineering. Nat Biotechnol 23(1):47-55

17. Bunyaratavej $P$, Wang $H-L$ (2001) Collagen membranes: a review. J Periodontol 72(2):215-229

18. Kim K-H, Jeong L, Park H-N, Shin S-Y, Park W-H, Lee S-C, Kim T-I, Park Y-J, Seol Y-J, Lee Y-M (2005) Biological efficacy of silk fibroin nanofiber membranes for guided bone regeneration. J Biotechnol 120(3):327-339

19. Kieswetter K, Schwartz Z, Hummert T, Cochran D, Simpson J, Dean D, Boyan B (1996) Surface roughness modulates the local production of growth factors and cytokines by osteoblast-like MG-63 cells. J Biomed Mater Res 32(1):55-63

20. Sui G, Yang X, Mei F, Hu X, Chen G, Deng X, Ryu S (2007) Poly-L-lactic acid/ hydroxyapatite hybrid membrane for bone tissue regeneration. J Biomed Mater Res A 82(2):445-454

21. Liu H-C, Lee I, Wang J-H, Yang S-H, Young T-H (2004) Preparation of PLLA membranes with different morphologies for culture of MG-63 cells. Biomaterials 25(18):4047-4056

22. Unger RE, Sartoris A, Peters K, Motta A, Migliaresi C, Kunkel M, Bulnheim U, Rychly J, James Kirkpatrick C (2007) Tissue-like self-assembly in cocultures of endothelial cells and osteoblasts and the formation of microcapillary-like structures on three-dimensional porous biomaterials. Biomaterials 28(27): 3965-3976

23. Vepari C, Kaplan DL (2007) Silk as a biomaterial. Prog Polym Sci 32(8): $991-1007$

24. Hämmerle $\mathrm{CH}$, Jung RE, Feloutzis $\mathrm{A}$ (2002) A systematic review of the survival of implants in bone sites augmented with barrier membranes (guided bone regeneration) in partially edentulous patients. J Clin Periodontol 29(s3):226-231

25. Minoura N, Tsukada M, Nagura M (1990) Physico-chemical properties of silk fibroin membrane as a biomaterial. Biomaterials 11(6):430-434

26. Berahim Z, Moharamzadeh K, Rawlinson A, Jowett AK (2011) Biologic interaction of three-dimensional periodontal fibroblast spheroids with collagen-based and synthetic membranes. J Periodontol 82(5):790-797

27. Carpio L, Loza J, Lynch S, Genco R (2000) Guided bone regeneration around endosseous implants with anorganic bovine bone mineral. A randomized controlled trial comparing bioabsorbable versus non-resorbable barriers. J Periodontol 71(11):1743-1749

28. Wang HL, Miyauchi M, Takata T (2002) Initial attachment of osteoblasts to various guided bone regeneration membranes: an in vitro study. Periodontal Res 37(5):340-344

29. Song J-Y, Kim S-G, Lee J-W, Chae W-S, Kweon H, Jo Y-Y, Lee K-G, Lee Y-C, Choi J-Y, Kim J-Y (2011) Accelerated healing with the use of a silk fibroin membrane for the guided bone regeneration technique. Oral Surg Oral Med Oral Pathol Oral Radiol Endod 112(6):e26-e33

30. Cai K, Yao K, Lin S, Yang Z, Li X, Xie H, Qing T, Gao L (2002) Poly (D, L-lactic acid) surfaces modified by silk fibroin: effects on the culture of osteoblast in vitro. Biomaterials 23(4):1153-1160

31. Chen W, Wang WW, Shi XZ, Chen N (2013) Evaluation of the biocompatibility and cell segregation performance of acellular dermal matrix as barrier membrane on guided tissue regeneration in vitro. Shanghai Kou Qiang Yi Xue 22(3):260-264

32. Kundu B, Rajkhowa R, Kundu SC, Wang X (2013) Silk fibroin biomaterials for tissue regenerations. Adv Drug Deliv Rev 65(4):457-470

33. Lee S-W, Kim S-G (2014) Membranes for the guided bone regeneration. Korean Association of Maxillofacial Plastic and Reconstructive Surgeons 36(6):239-246

34. Jang E-S, Park J-W, Kweon H, Lee K-G, Kang S-W, Baek D-H, Choi J-Y, Kim S-G (2010) Restoration of peri-implant defects in immediate implant installations by Choukroun platelet-rich fibrin and silk fibroin powder combination graft. Oral Surg Oral Med Oral Pathol Oral Radiol Endod 109(6):831-836

35. Sofia S, McCarthy MB, Gronowicz G, Kaplan DL (2001) Functionalized silk-based biomaterials for bone formation. J Biomed Mater Res 54(1):139-148 